\title{
功能整合与战略规划：高速铁路车站建设与城市发展 研究评述
}

\author{
Functional Integration and Strategic Planning: A Review of Studies on High-speed Rail Station and \\ Urban Development
}

杨跃龙 韩笋生

Yang Yuelong, Han Sunsheng

\begin{abstract}
摘要: 伴随高速铁路建设发展, 高速铁路车站在城 市中的作用逐渐显现并日益多样化。国内外经验表 明, 高速铁路车站对站点区域的土地利用、城市品 质产生影响, 并会影响其所在城市的经济发展和空 间形态。注重功能整合、规划过程和多方参与的战 略性规划有助于实现高速铁路车站对城市发展的积 极影响。

Abstract: In parallel with the rapid development of Highspeed Rail networks around the world, High-speed Rail stations have begun to play an increasingly active role in cities. Experiences from Europe, Japan and China demonstrate that High-speed Rail stations shape the land use, urban quality, economic development and spatial layout of cities where they sit. By emphasising functional integration and a participatory planning process, strategic planning helps maximize the positive influence of Highspeed Rail stations on urban development.
\end{abstract}

关键词: 高速铁路车站; 城市发展; 城市空间变化; 战略规划; 车站功能

Keywords: High-speed Rail Station; Urban Development; Urban Spatial Change; Strategic Planning; Station Function

作者：杨跃龙, 澳大利亚墨尔本大学建筑与规划学院, 博士研究生。yuelongy@student.unimelb.edu.au 韩笋生, 澳大利亚墨尔本大学建筑与规划学院, 教授，博士生导师。sshan@unimelb.edu.au

\section{引言}

以 1964 年连接日本东京与大阪的东海道“新干线”建成通车为标 志, 现代高速铁路（简称 “高铁”）至今已历经半个多世纪的发展。进 人 21 世纪以来, 高铁在世界范围内快速建设 ${ }^{[1]}$ (图 1)。目前, 全球已 有 16 个国家和地区使用高铁 ${ }^{[2]}$ 。作为大型快速交通系统, 高铁在很大程 度上缩短了城市间的通行时间, 促进了区域的融合和城市体系的重建 ${ }^{[3]}$, 而其对区域和城市空间发展的影响在不同尺度上也均有显现 ${ }^{[47]}$ 。依据空间 尺度不同, 既有高铁研究可分为区域层面、城际层面、城市层面和车站层 面。在区域层面, 现有文献主要关注高铁的空间发展效应 : 中心区域与外围 区域的发展分化、设站区域和非设站区域的发展差距、经济要素在高铁沿线 的分布等 ${ }^{[8.9]}$ 。在城际层面, 高铁不仅改变了城市间的绝对和相对交通可达 性, 还影响了个人与企业的区位选择, 带来了新的城市间关系 ${ }^{[1,10-11]}$ 。在城市 层面, 高铁对城市系统重构和空间形态重塑扮演着催化剂 (catalyst) 和促进 器 (facilitator) 的作用, 并为城市经济发展提供机遇 ${ }^{[12-13]}$ 。在车站层面, 高铁 与站区的土地利用、房地产开发、城市空间形态、城市品质等紧密相关 ${ }^{[1416]}$ 。

随着高铁在世界范围内的快速发展, 有关高铁站区建成环境变化这一微 观尺度的研究日益增多, 相关案例素材不断丰富, 在高铁建设与区域经济和 空间变化的研究之外, 逐步形成了一个新的研究领域和关注热点。国内已有 学者从高铁枢纽建设与城市发展 ${ }^{[17]}$ 、站区圈层用地布局、人口集聚、产业 布局 ${ }^{[18]}$ 及城市空间演化 ${ }^{[19]}$ 等方面对相关文献进行了回顾。但对车站多重属 性、多种区位、多面影响、多方参与及由此所决定的功能整合和战略规划的 梳理有所欠缺。本文以此思路回顾国内外相关文献对高铁车站属性、区位及 其在站区和所在城市两个微观尺度上的影响等方面的研究发现：(1) 高铁车 站多重属性决定其在城市建设中体现更多功能；（2）车站区位选择对城市带 来不同影响; (3) 在站区层面, 车站及相关建设项目将影响土地利用和城市 品质；（4）在所在城市层面, 还将对经济发展和空间形态产生影响；（5）基 于车站的多功能属性和多方面影响, 在高铁车站项目规划建设时, 应注重规 划尺度的细分和综合, 注重规划过程和多方利益协调, 注重多功能的战略性 规划。 


\section{1 对高铁车站的再认识}

\section{1 车站相关城市建设项目的兴起}

高铁的蓬勃发展带动了许多与其相关的大型城市建设项 目的兴起, 越来越多的地方政府将高铁车站建设与车站周边 区域甚至是更广范围内的城市更新与发展项目相结合。是谁 以及何种原因驱动了这一现象? 既有文献探究了四个方面的 原因。首先，全球化发展背景下，城市间对资本、技术、人 力的争夺逐渐加剧, 城市管理逐渐向城市运营转变。高铁的 高品质及其车站所塑造的高端形象正好契合了城市运营者对 提升城市竞争力的追求。他们希望借此吸引更多的产业在本 地聚集 ${ }^{[8,13,20]}$ 。其次, 随着城市化发展, 人们开始担忧城市 蔓延及依赖汽车通勤的城市化发展模式, 从而出现了大城市 的去中心化发展及交通和土地利用规划由汽车主导模式向高 密度、重步行和以公共交通为导向模式的转变 ${ }^{[21]}$ 。再次, 国 家铁路公司的私有化导致铁路公司出现新的逐利模式。它们 一方面积极营销高铁, 一方面竭力实现自持土地资产的价值 提升, 同时不断扩大与当地政府的合作, 以通过参与城市建 设项目来实现最大盈利 ${ }^{[20]}$ 。最后, 旧有火车站往往处在城市 中心且很多车站周边土地利用效率低下, 高铁车站建设可以 为改善旧站环境提供绝佳机遇 ${ }^{[8]}$ 。

\section{2 节点与场所、流空间与场所空间：车站的多重属性}

对车站属性的认识从根本上决定了对站区进行规划时所 秉持的原则, 以及车站在建成之后所担当的角色和发挥的作 用。越来越多的研究强调车站同时具备节点和场所属性 : 它 们既是交通网络中的节点, 也是城市中的活动场所; 且两种 属性需要相互协调, 否则将诱发车站区域的再度开发 ${ }^{[6,22-23]}$ 。
罗伊瑟等 (Reusser et al.) 以 “节点一场所” 模型为基础, 测 度了瑞士各火车站节点与场所属性的协调性, 发现它们并非 呈线性关系, 表明在二者之外还可能存在其他维度的属性影 响着站区的协调发展 ${ }^{[24]}$ 。在 “节点一场所” 维度之上, 若加 人“区位一网络” 维度, 则可将车站属性进一步细分为四种: 连接交点 (connector)、交通节点 (transportation node)、会 面场所 (meeting place) 及城市中心 (urban centre), 这四种 属性可能相互协调和冲突, 且在不同的规划开发阶段发挥不 同作用 ${ }^{[25]}$ 。国内研究也开始关注车站地区交通和城市功能的 平衡发展, 并指出在规划设计时应打破行业局限, 谋求区域 和城市发展的协调 ${ }^{[26-28]}$ 。

基于卡斯特 (Castells) 有关 “流空间”（space of flow） 和 “场所空间” (space of place) 的理论 ${ }^{[29]}$, 作为主要交通走 廊节点的高铁站还体现了流空间和场所空间的交融：一方面 高铁站承载着来自国际的资本、技术、人才等的流动, 另一 方面它又是当地社会经济的物质体现。社会、多方参与者与 空间之间的相互作用, 最终形成了车站区域的现状, 而公开、 公平、透明的规划过程及政府管理方式的革新有助于避免流 空间对城市原有环境和活力的破坏 ${ }^{[30]}$ 。高铁站区规划的核心 在于车站不能仅被视作人们换乘的节点, 它们同时还是集聚 各种高价值经济社会活动的空间聚点, 且将对城市的发展带 来积极影响 ${ }^{[31]}$ 。高铁车站既是城市内部多种经济社会活动集 聚的场所, 也是城市外部资源汇聚的节点。资金、技术、信息、 人才通过诸如高铁、信息技术、通信技术等基础设施在城市 间、区域间和国际间流动, 超越了地域的尺度, 影响和改变 着城市发展和人们的生活; 同时, 为了吸引、竞争这些有限 的资源, 管理者和规划者又在调整和改变着他们的规划管理 尺度, 以不断改善当地环境来凝聚这些资源 ${ }^{[32]}$ 。高铁站正是

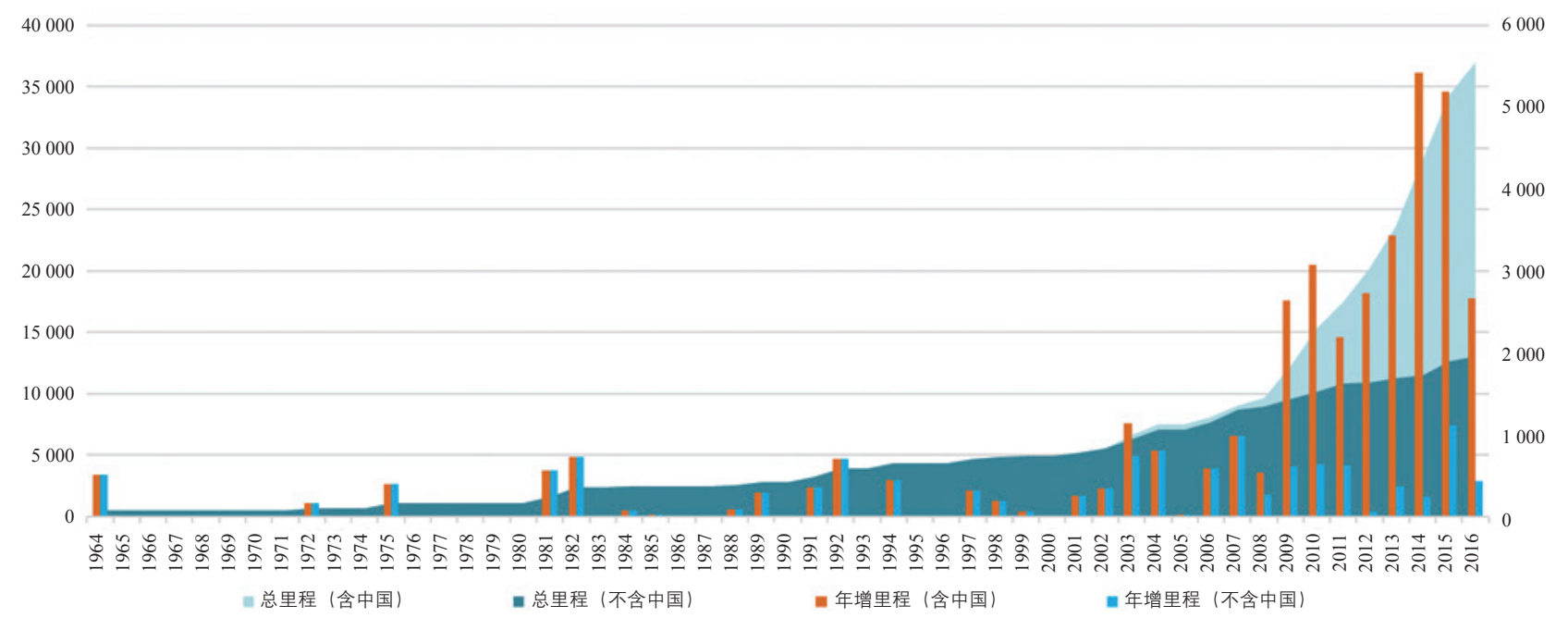

图 1 1964一2016 年世界高铁建设规模（单位 : km )

资料来源 : 国际铁路联盟客运部 (UIC Passenger Department) 
这种流动的资源和演进的社会相互交织的节点。所以, 对站 区的规划也应注重从国家、区域、城市等多种尺度予以细化 分析和整合。只有对多维度、多层次、多参与方的综合考虑 和长远规划, 才可能实现车站区域的协调可持续发展。

\section{3 巩固旧城还是发展新区 : 车站的多种区位选择}

高铁车站可以设在城市的不同位置, 并将影响当地交通 可达性和空间发展 ${ }^{[33]}$ 。在欧洲, 大都市常将高铁站设在传统 的中心区域, 如荷兰阿姆斯特丹中央站、德国柏林中央车站 等, 但也有一些在主城边缘设站, 如法国里尔欧洲站等。在 中小城市或人口密度较低的地区, 设在城市边缘甚至边远地 区的高铁站也很常见 ${ }^{[34]}$ 。在日本, 情况也很类似, 如东京站、 名古屋站、京都站都位于原有的城市中心 ; 而新横滨站和新 大阪站却设在距市中心较远的地方 ${ }^{[35}$ 。我国高铁站选址通常 位于城市边缘, 以期对城市郊区化发展产生促进作用 ${ }^{[36]}$ 。但 实证经验表明, 车站距城市中心距离影响着高铁站区的开发 建设, 且城市规模越大、距离指数越小, 开发量越大 ${ }^{[37]}$ 。

不同区位的车站将发挥不同的作用, 关键是规划者能否 将其选址在最优的位置从而将最亟须体现的功能最大化。依 据车站区位不同, 霍尔 (Hall) 指出高铁会带来三种影响 : 当车站设在传统的商业中心或其附近时, 高铁站可以帮助提 高和巩固 $\mathrm{CBD}$ 区域的商业吸引力; 当设在城市边缘时, 车 站可以帮助建设新的城市副中心; 当设在城市边远地区时, 则可利用车站建设发展城市新区 ${ }^{[38]}$ 。梅内德斯等 (Menéndez et al.) 指出, 在主城边缘设站是最理想的选择, 因为那里既 有足够的开发空间, 又能有效衔接旧城区, 同时还能吸引来 自城市内外的商业活动 ${ }^{[39 !}$ 。车站的多重属性决定了在对其进 行规划时, 除考虑交通因素外, 还应注重城市发展其他方面 的因素。而针对我国高铁车站的复杂性, 王缉宪和林辰辉认 为, 在对其进行规划和分析时, 还需将车站区位与功能类型 （枢纽站、中途站、终端站、接驳站）等综合考虑 ${ }^{[40]}$ 。最初 仅担当快速连接主要城市交通任务的高铁, 逐渐承担了越来 越多的功能期望。城市管理者和规划者也应注重将站区的交 通规划与整个城市的发展规划进行战略性的整合, 以一种综 合的战略视角来协调和整合车站各种功能的实现。

\section{2 车站建设与站区开发}

\section{1 多元用途与集约发展 : 站区土地利用变化}

高铁车站建设对站区的影响, 首先体现在土地利用变化 上。沈爆等对西班牙马德里阿托查站的研究发现, 高铁带来 的区域可达性提升导致了站区城市建设用地的增加。虽然高 铁并非唯一影响因素, 但其作用十分显著 ${ }^{[41]}$ 。舒茨和波尔 (Schütz \& Plo) 提出的 “三个发展区” 圈层结构模型指出,
车站周边的土地利用将呈现圈层式发展 ${ }^{[12]}$ 。而郝之颖对我国 车站地区的研究认为, 第一圈层与车站关联最紧密, 用地空 间受枢纽影响最大; 第二圈层包括办公、商务、居住等, 规 划时应注重土地经济性的培育; 第三圈层是间接催化区域, 重在保持站区与城市整体布局的协调 ${ }^{[2]}$ 。

车站建设会带动站区土地利用方式发生转变, 且多种用 途都会出现。写字楼用地在欧洲高铁站区最为常见。事实上, 无论最初的规划如何追求用地多样性, 站区开发的高成本和 写字楼物业的高收益都会驱使人们将写字楼作为首选, 并减 少其他用地比例。在荷兰的阿姆斯特丹和乌得勒支, 写字楼 用地不仅自身实现盈利, 还使整个开发项目变得可行 ${ }^{[22]}$ 。在 我国, 很多车站地区也被规划为 “高铁商务区”, 且规模很

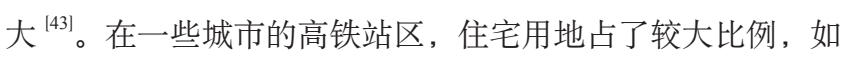
西班牙的萨拉戈萨和巴拉多利德 ${ }^{[34]}$ 。在法国旺多姆这一现 象也很明显, 因为高铁的修建会使车站地区距巴黎的交通时 间大幅缩减为 40 分钟, 吸引了大量的巴黎上班族 ${ }^{[44]}$ 。此外, 一些城市还在高铁站附近规划了工业园区、物流设施和服务 业用地 ${ }^{[34]}$, 如萨拉戈萨的物流平台 ${ }^{[45]}$ 。在我国, 一些车站 也规划了工业用地, 如德州 ${ }^{[46]}$ 。

目前, 大多数高铁站区规划都提倡多功能土地利用, 因 为这是影响站区活力、吸引力和安全性的重要因素 ${ }^{[6,47]}$ 。卡 斯塞塔和帕利亚拉（Cascetta \& Pagliara）强调车站周边土 地利用方式的协调, 并指出通过增加建筑密度、调整站区 重要公众建筑或标志性建筑的选址可以实现站区用地的协 调 ${ }^{[48]}$ 。城市经济结构重组、功能分区及城际交通革新, 导 致了土地的多功能利用。在土地资源相对稀缺的一些关键 地点 (车站), 这种多用途利用尤其明显 ${ }^{[49]}$ 。土地多功能利 用可以带来协调性优势, 同时有利于促进经济活力、展现 城市品质 ${ }^{[50]}$ 。深圳北站、福田站等在规划建设时也在这方 面做了有益尝试 ${ }^{[51]}$ 。

\section{2 城市形象与空间营造: 站区城市品质提升}

新技术、新建筑材料及现代化设计理念的应用, 在很大 程度上提升了站区的城市形象和品质。法国里尔欧洲站、荷 兰阿姆斯特丹南站和鹿特丹中心站都是很好的例证 ${ }^{[16]}$ 。意大 利坎帕尼亚区域轨道交通网的建设说明, 车站建设尤其是一 些高质量的新站建设可以提升周边地区的城市品质 ${ }^{[48]}$ 。高铁 站区的价值不仅体现在增加远距离交通的可达性上, 同样重 要的是, 这些项目通过建设高级别的地标性建筑, 能够重塑 车站地区形象, 从而整体提升城市品质 ${ }^{200}$ 。国内对于这方面 的专门研究较少, 但在一些车站的规划实践中, 对城市品质 的追求和空间的营造已有所体现, 如南京南站 ${ }^{[52]}$ 。

除提升交通可达性带动当地经济发展之外, 新的高规 
格的车站建设所带来的城市形象提升也有助于当地的经济发 展。特里普 (Trip) 认为, 高铁发展激发了欧洲城市之间以 修建高铁来促进经济和城市形象的竞争, 这种竞争与他们积 极申办奥运会和世博会并无差别 ${ }^{[15]}$ 。具体来说, 城市品质体 现在城市结构、建筑、功能多样性、公共区域品质等方面。 同时承载城市商业和住宅功能的高铁站区尤其影响着一个城 市的品质。在车站工程规划设计中仅关注房地产开发、基础 设施建设和交通衔接是不够的, 因为一些在工程上难以体现 的因素也影响着车站区域的城市品质。以政府为主导、多方 参与的、弹性的规划过程则有助于保证城市品质的提升 ${ }^{[16]}$ 。 德国 “斯图加特 21 ” 项目所造成的公众强烈反对现象说明, 高铁车站不是一个简单的场所, 而是城市生活的场所, 是城 市重要的标志和象征, 在城市环境中起着多方面的重要作用。 高铁车站工程的规划和建设要注重过程的公开透明和公众参 与, 要将城市品质和城市历史因素考虑进去 ${ }^{[53]}$ 。

\section{3 车站建设与所在城市发展}

\section{1 经济增长}

两方面的理论解释了高铁车站建设对经济的影响。可达 性理论认为, 高铁能降低城市的一般交通成本 (generalised transportation cost), 提升居民的最大可接受交通距离（the maximum acceptable transportation distance), 从而享受到更 多的外部资源和福利, 带来更大的经济增长 ${ }^{[12]}$ 。城市品质理 论认为, 在可达性提升之外, 高速列车及车站所带来的城市 形象提升也能够增强一个城市的竞争力。这是因为城市的经 济竞争力源于创新力、创造力和知识溢出, 而这些都与高技 能人才和高教育群体的集聚有关。较高的城市品质可以有效 吸引这些人才。因此, 高铁站区规划和开发将有助于提升城 市品质进而提升城市竞争力 ${ }^{[15]}$ 。

高铁车站建设并非带动经济发展的充分条件, 若想实现 经济增长和城市更新还需满足一些前提条件, 否则会引致负 面效应 ${ }^{[12]}$, 如城市空间发展不平衡 ${ }^{[54]}$ 、企业外迁 ${ }^{[55]}$ 等。一 些城市的车站建设带动了当地经济发展, 而另一些则不然。 在日本, 新干线车站建设等项目刺激了东京中心城区的经济 发展 ${ }^{[56]}$, 并对区域产业选址产生积极影响 ${ }^{[57]}$ 。在法国, 里 尔欧洲站开发建设对当地的房地产市场、住房市场和就业市 场产生了很大影响, 吸引了来自里尔之外的消费群体。同时 伴随着政府的鼓励政策, 车站建设实现了将中低收人住房需 求从里尔市区向都市低价地区分流的目的 ${ }^{[58]}$ 。然而, 在 “巴 黎一里昂” 高铁沿线上的三个新站建设中, 只有里昂的帕尔 迪厄站有明显的发展 ${ }^{[59]}$ 。德国柏林也没有迎来高铁站周边的 更新和发展, 而大量的投资仅给当地房地产市场和整体经济 带来了微弱的影响 ${ }^{[60]}$ 。英国肯特的阿什福德站也没有明显的
发展, 而距伦敦 $16 \mathrm{~km}$ 之外的艾贝斯费特站仅仅修建了停车 设施 ${ }^{[61]}$ 。所谓 “高铁新城” 的发展并不一定与高铁车站建设 相关一一法国高铁建设并没有伴随着新城的建设, 相反, 那 些有新城建设和发展的地区却没有修建高铁 ${ }^{[61]}$ 。很多城市预 期通过高铁站的修建来带动城市发展和转型, 但一些不切实 际的、盲目乐观的大型规划受经济周期等外部环境的影响难 以实现预期的效果 ${ }^{[45]}$ 。

关于高铁车站建设是否带来当地经济发展的实证检验 仍然存在分歧, 但学者们逐步认同的是, 在条件满足的前 提下这种带动作用有可能实现。因此, 现在的问题是哪些 条件能够促进这种积极作用的实现。洛凯托 - 赛德里斯等 (Loukaitou-Sideris et al.) 通过对 27 位专家开展的德尔菲分 析发现, 车站选址, 以城市设计的理念来指导站区的空间设 计, 与其他多种交通方式相连, 有支撑性的土地利用和分区 政策, 以及兼顾多方利益的规划开发过程等, 都有助于实现 高铁站区的成功开发 ${ }^{[62]}$ 。林辰辉认为, 城市发展水平、城市 空间、车站自身、车站可达性、站区现状等均影响站区开发, 而所在城市的经济发展水平、高铁枢纽本身较高的客流量及 与城市中心的空间和时间距离是影响站区开发效果的主要因 素 ${ }^{[63]}$ 。与此相近, 王缉宪、林辰辉认为, 车站、车站所在城 市、城市所在区域三个地理层面的多方因素决定了我国站区 开发的成败, 并据此构建了评价车站发展建设的指标体系 ${ }^{[40]}$ 。 国内外实践表明, 城市开发与更新非常复杂, 因为涉及开发 过程中的诸多不确定性因素, 将面临不同层级地方政府间的 竞争 ${ }^{[6]}$ 。若想通过交通建设来带动城市发展, 需要同时满足 经济环境、资金投人以及政策制度等前提条件 ${ }^{[64]}$ 。

\section{2 空间形态变化}

通过改变站区可达性和增加投资，车站建设还将对城市 的空间形态产生影响。以里尔、布鲁塞尔、伦敦、马德里和 鹿特丹为例, 霍尔指出, 通过带动站区发展, 高铁建设进一 步加强了中央商务区的地位 ${ }^{[38]}$ 。不管是建在城市中心还是郊 区, 车站建设均可成为城市功能和形态调整的良好机遇。许 多西班牙的中小城市利用高铁站建设, 同时启动了城市更新 和改造项目, 改变了旧有铁路对城市功能和形态的分割, 实 现了城市与高铁建设的协调发展 ${ }^{[65]}$ 。而当地政府不同的管理 战略 (如, 将车站与其他交通方式相连、减弱车站对城市的 分裂作用、建设车站的新城市形象等）带来了法国斯特拉斯 堡、荷兰阿纳姆、英国斯特拉特福德、西班牙昆卡、德国卡 塞尔、比利时列日等城市高铁站区的更新和发展 ${ }^{[66]}$ 。我国高 铁车站多为近年建设开发, 其对所在城市空间形态的影响仍 未完全显现, 但从其规划内容看, 较多的车站区域都被定位 为综合发展的城市新区或新城，而商务金融服务、商业休闲、 
房地产等为主要产业类型 ${ }^{[52]}$ 。

新车站的建设可以改变原有的城市发展重心, 并加速城 市边远地区的城镇化发展 ${ }^{[67]}$ 。同时, 这种建设还可能将城市 从单中心向多中心模式转变 ${ }^{[68]}$ 。高铁最初的建设目的是连接 各大都市区, 然而随着建设规模的增加, 一些大都市腹地中 的小城市或城镇也连接进了高铁网络。这便有可能形成一种 新的都市通勤模式, 从而扩大都市区的影响范围。伦敦和马 德里等大都市周边的高铁站区逐渐集聚了一些产业和人口, 并起到了去中心化的作用。因此, 高铁规划应该考虑相关的 地域因素、基础设施及服务, 不能仅将高铁规划视作一种交 通规划, 而应将其纳人城市规划当中 ${ }^{[69]}$, 以一种更加综合的 视角, 战略性的进行协调。

\section{4 对用地功能及规划过程的战略整合}

近半个多世纪的实践表明, 高铁车站及相关城市建设项 目的确可以对当地的土地利用、空间形态、企业及居民选址、 城市品质等产生影响。在对高铁车站这种大规模、多投人、 长周期的项目进行规划时, 应该综合考量, 站在战略高度谋 划功能布局、协调各参与方的利益。高铁对城市的影响很大 程度上取决于当地各参与者组织利用高铁所带来的资源的能 力。如果缺少对规划建设过程有效的内外部管理, 缺乏对各 参与者的有序组织, 开发项目将不能在最大程度上带动相关 区域的发展, 也会导致各层级利益相关者的摩擦与冲突, 最 终影响建设效果 ${ }^{[70]}$ 。

很多站区规划都是一种对多元功能的整合。通过对欧 洲数百个车站项目的总结和分析, 皮特斯和诺维 (Peters \& Novy) 归纳了车站周边项目规划开发的四种类型：战略型超 大项目、车站重建项目、交通类项目、城市开发型项目, 并 指出一些项目已经超越了交通或城市建设层面, 而上升为一 种长远的战略性工程 ${ }^{[20]}$ 。林辰辉和马璇根据产业功能不同将 我国典型高铁车站归纳为四类: 均衡型、商务功能主导型、 居住功能主导型、商贸功能主导型 ${ }^{[71]}$ 。而现实中很多项目其 实是多种类型的综合, 虽各有侧重, 但绝非单一功能的规划。 因此, 规划师在规划伊始就应该明确项目多层次目标并在规 划及建设过程中竭力谋求多种功能的整合和协调。

多功能的整合必然涉及参与方的多元利益、并要求公 共部门内部及其与私人部门之间加强协调。贝尔托利尼 （Bertolini）以荷兰乌得勒支城市工程、英国英王十字车站 和法国里尔欧洲站建设为例, 对比分析指出, 政府与私人 部门等的参与及联合, 以及这种公私联合体与其他各方的 有效沟通, 是项目成功的关键 ${ }^{[6]}$ 。尤其对于车站设在城市边 缘的中小城市, 政府有效的介人至关重要。因为能否克服 交通可达性差、周边商业设施差等困难关乎这一车站的成
败。而大多数类似规划尚都缺乏不同公共管理部门间的协 调以及政府与私人部门间的合作 ${ }^{[34]}$ 。空间规划应该涉及更 广阔的领域, 需要整合不同功能类型的项目, 这不仅需要 提升空间协调度、保证规划的可行和现实, 还要寻求公共 与私人部门的合作, 满足各方利益 ${ }^{[49]}$ 。车站的物理形态仅 是站区吸引商业活动、得到繁荣发展的诸多因素中的一个 方面。站区的规划和开发需要同时兼顾三个维度: 城市本身、 规划与开发过程、制度因素。保证各参与主体的有效沟通 是高铁站区开发成功的关键 ${ }^{[23]}$ 。

协调多元利益需要关注规划过程。由于多年较慢的决策 过程和随之而来的市场投机, 比利时布鲁塞尔高铁站的建设 危及了城市原有的社会和经济肌理, 影响了车站地区的城市 品质和环境质量。而综合不同空间尺度（地方、区域、国家） 的战略性规划不但协调不同层级政府的规划政策诉求, 还可 以帮助解决 “以市场为导向” 和 “积极政府干预” 间的矛 盾; 同时, 应建立一种公平、公开、透明的决策程序以使地 方的声音得到表达 ${ }^{[30]}$ 。应该注重规划的过程, 以及各种权力 关系在其中的变化和重新组合 ${ }^{[22]}$ 。规划时对某些方面的侧重 往往与价值观和偏好相关, 因此, 高铁站区的规划应该以规 划过程为导向, 尽量增加多方参与, 保证沟通有效, 实现协 商、合作式的规划 ${ }^{[24]}$ 。

\section{5 启示}

首先, 我国的高铁车站规划与开发须借鉴国际经验。注 重规划尺度的细分和整合, 注重规划过程和多方参与, 注重 多种功能的战略性整合。具体来讲, 一是明晰车站的多重属 性, 明确车站区域开发需实现的主要功能和辅助功能, 在站 点选择、土地利用规划、产业布局、交通衔接等方面有所偏 重和整合。二是把握城市发展的脉络和方向, 注重土地多功 能节约集约利用, 注重与多种交通方式的衔接。三是注重城 市品质的创建, 提倡以人为本, 关注城市社会经济活动, 注 重对城市历史元素的保护和新品质的创造。

其次, 认清我国城市发展阶段和经济水平与国外发达国 家的差异。日本和欧洲等已经建设运行高铁的多数国家均实 现了较高的城镇化, 且经济结构也已转为以第三产业为主, 而我国与此则有很大不同。也正因此, 我国高铁及其车站建 设将承载更多的任务, 并将与城镇化、城市群建设、产业结 构调整等政策相关。具体到站区的规划建设, 则应该注重交 通功能与居住、商业功能的协调, 注重产业的分布和城市功 能分区, 注重从城市、区域、国家等多种尺度考量车站的选 址、功能分区、产业布局和交通衔接等。

最后, 以长远性、战略性思维统筹站区规划开发。国外 经验表明, 车站区域的成功开发依赖经济环境、资金投人和 
政策制度三方面的积极条件, 缺一不可。在车站区域规划开 发时应当认清当地客观条件和高铁有可能带来的正面影响, 避免盲目乐观而制定宏大却缺乏支撑的开发规划，应该注重 细节（慎重选址、合理分区、控制规模、完善与其他交通方 式衔接等）尽力创造有益条件, 将车站带动作用实现的可能 最大化。长远规划并非体现在空间预留的提前量, 更非土地 规模的一味扩大, 它是一种战略性的对多种维度的统筹, 需 要对多种资源、多方参与者、多种功能的系统性整合。因此, 应该注重规划开发过程和多方利益表达，结合区域和城市特 点因地制宜的协调各种资源, 为实现高铁车站建设与城市发 展创造条件。UPI

\section{参考文献}

[1] GARMEndia M, RiBAlAYGUA C, UREÑA J M. High speed rail: implication for cities[J]. Cities, 2012, 29: S26-S31.

[2] UIC PASSENGER DEPARTMENT. High speed lines in the world[DB/ OL]. (2017-04-01) [2017-05-23]. http://uic.org/IMG/pdf/high_speed_lines_ in_the_world.pdf

[3] YIN M, BERTOLINI L, DUAN J. The effects of the high-speed railway on urban development: International experience and potential implications for China[J]. Progress in planning, 2015, 98: 1-52.

[4] BLUM U, HAYNES K E, KARLSSON C. Introduction to the special issue the regional and urban effects of high-speed trains[J]. The annals of regional science, 1997, 31(1): 1-20.

[5] CHEN C L, HALL P. The impacts of high-speed trains on British economic geography: a study of the UK's Inter City 125/225 and its effects[]]. Journal of transport geography, 2011, 19(4): 689-704.

[6] BERTOLINI L. Nodes and places: complexities of railway station redevelopment[]]. European planning studies, 1996, 4(3): 331-345.

[7] BRUINSMA F. The impact of railway station development on urban dynamics: a review of the Amsterdam South Axis project[J]. Built environment, 2009, 35(1): 107-121.

[8] UREÑA J M, MENERAULT P, GARMENDIA M. The high-speed rail challenge for big intermediate cities: a national, regional and local perspective[J]. Cities, 2009, 26(5): 266-279.

[9] GIVONI M. Development and impact of the modern high-speed train: a review[J]. Transport reviews, 2006, 26(5): 593-611.

[10] CHEN C L, HALL P. The wider spatial-economic impacts of high-speed trains: a comparative case study of Manchester and Lille sub-regions[J]. Journal of transport geography, 2012, 24: 89-110.

[11] LEVINSON D M. Accessibility impacts of high-speed rail[J]. Journal of transport geography, 2012, 22: 288-291.

[12] POL P M J. The economic impact of the high-speed train on urban regions $[R]$. European regional Science Association, 2003.

[13] PRIEMUS H. HST-railway stations as dynamic nodes in urban networks[C]. 3rd CPN Conference Proceeding, 2006, 5: 101-121.

[14] SANDS B. The development effects of high-speed rail stations and implications for California[J]. Built environment, 1993: 257-284.

[15] TRIP J J. What makes a city? planning for 'quality of place': the case of high-speed train station area development[M]. IOS press, 2007.

[16] TRIP J J. Urban quality in high-speed train station area redevelopment: the cases of Amsterdam Zuidas and Rotterdam Centraal[J]. Planning, practice \& research, 2008, 23(3): 383-401.

[17] 林辰辉, 马林, 杨少辉. 高铁及枢纽建设对城市发展影响的研究综述 [C]. 2010 年中国大城市交通规划研讨会一中国城市交通规划年会论文 集, 2010 .
[18] 王丽, 刘可文, 曹有挥。国内外高铁站区空间结构研究进展及启示 [J]. 经济地理, 2016(8): 120-126.

[19] 洪世键, 姚超. 高速铁路站点与城市空间演化: 述评与反思 [J]. 国际城 市规划, 2016(2): 84-89.

[20] PETERS D, NOVY J. Train station area development mega-projects in Europe: towards a typology[J]. Built environment, 2012, 38(1): 12-30.

[21] PEEK G J, BERTOLINI L, DE JONGE H. Gaining insight in the development potential of station areas: a decade of node-place modelling in the Netherlands[]]. Planning, practice \& research, 2006, 21(4): 443-462.

[22] BERTOLINI L. Station area redevelopment in five European countries: an international perspective on a complex planning challenge[]]. International planning studies, 1998, 3(2): 163-184.

[23] BERTOLINI L. Planning in the borderless city: a conceptualisation and an application to the case of station area redevelopment[J]. Town planning review, 2000, 71(4): 455-475.

[24] REUSSER D E, LOUKOPOULOS P, STAUFFACHER M, et al. Classifying railway stations for sustainable transitions-balancing node and place functions[]]. Journal of transport geography, 2008, 16(3): 191-202.

[25] PEEK G J, LOUW E. Integrated rail and land use investment as a multidisciplinary challenge[J]. Planning, practice \& research, 2008, 23(3): 341361.

[26] 郑德高, 杜宝东. 寻求节点交通价值与城市功能价值的平衡一一探讨 国内外高铁车站与机场等交通枢纽地区发展的理论与实践 [J]. 国际城 市规划, 2017, 22(1): 72-76.

[27] 段进. 国家大型基础设施建设与城市空间发展应对一以高铁与城际 综合交通枢纽为例 [J]. 城市规划学刊, 2009(1): 33-37.

[28] 殷铭, 汤晋, 段进. 站点地区开发与城市空间的协同发展 [J]. 国际城市 规划, 2013(3): 70-77.

[29] CASTELLS M. The information age: economy, society and culture. Vol. 2, The power of identity[M]. Blackwell, 1997.

[30] ALBRECHTS L, COPPENS T. Megacorridors: striking a balance between the space of flows and the space of places[J]. Journal of transport geography, 2003, 11(3): 215-224.

[31] BRUINSMA F, PELS E, PRIEMUS H, et al. Railway development: impacts on urban dynamics[M]. Springer Science \& Business Media, 2007.

[32] BRENNER N. New state spaces: urban governance and the rescaling of statehood[M]. Oxford and New York: Oxford University Press, 2004.

[33] FACCHINETTI-MANNONE V. Location of high speed rail stations in French medium-size city and their mobility and territorial implications $[\mathrm{C} /$ OL]. City futures 09. 2009[2017-05-23]. http://www. cityfutures2009. com/ papers_viernes. htm.

[34] BELLET C. Peripheral high-speed rail stations in Spain[J]. The open transportation journal, 2016, 10(1): 45-56.

[35] SMITH R. The Japanese Shinkansen: catalyst for the renaissance of rail[J]. The journal of transport history, 2003, 24(2): 222-237.

[36] 于涛, 陈昭, 朱鹏宇. 高铁驱动中国城市郊区化的特征与机制研究— 以京沪高铁为例 [J]. 地理科学, 2016, 32(9): 1041-1046.

[37] 赵倩, 陈国伟。区位对高铁车站周边地区开发的影响 [J]. 城市交通, 2015, 13(3): 17-23.

[38] HALL P. Magic carpets and seamless webs: opportunities and constraints for high-speed trains in Europe[J]. Built environment, 2009, 35(1): 59-69.

[39] MENÉNDEZ J M, GUIRAO B, CORONADO J M, et al. New high-speed rail lines and small cities: locating the station[J]. WIT transactions on ecology and the environment, 2002, 54: 811-820.

[40] 王缉宪, 林辰辉. 高速铁路对城市空间演变的影响: 基于中国特征的 分析思路 [J]. 国际城市规划, 2011(1): 16-23.

[41] SHEN Y, E SILVA J A, MARTÍNEZ L M. Assessing high-speed rail's impacts on land cover change in large urban areas based on spatial mixed logit methods: a case study of Madrid Atocha railway station from 1990 to 2006[J]. Journal of transport geography, 2014, 41: 184-196.

[42] 郝之颖。高速铁路站场地区空间规划 [J]. 城市交通, 2008, 6(5): 48-52 
[43] 郑德高, 张晋庆. 高铁综合交通枢纽商务区规划研究——上海虹桥 枢纽与嘉兴南站地区规划为例 [J]. 规划师, 2011, 27(10): 34-38.

[44] RIETVELD P, BRUINSMA F R, VAN DELFT H T. Economic impacts of high speed trains. Experiences in Japan and France: expectations in the Netherlands[R]. VU University Amsterdam, Faculty of Economics, Business Administration and Econometrics, 2001.

[45] BELLET C, SANTOS GANGES L. The high-speed rail project as an urban redevelopment tool[J]. Belgeo, 2016(3): 1-18.

[46] 王兰. 高速铁路对城市空间影响的研究框架及实证 [J]. 规划师, 2011, 27(7): 13-19.

[47] DE GRAAFF T, DE GROOT H L F, RODENBURG C A, et al. The WTP for facilities at the Amsterdam Zuidas[J]. Environment and planning a, 2007, 39(9): 2099-2118.

[48] CASCETTA E, PAGLIARA F. Integrated railways-based policies: the regional metro system (RMS) project of Naples and Campania[J]. Transport policy, 2008, 15(2): 81-93.

[49] PRIEMUS H, HALL P. Multifunctional urban planning of mega-cityregions[J]. Built environment, 2004, 30(4): 338-349.

[50] RODENBURG C A, NIJKAMP P. Multifunctional land use in the city: a typological overview[J]. Built environment, 2004, 30(4): 274-288.

[51] 宗传苓, 谭国威, 张晓春. 基于城市发展战略的深圳高铁枢纽规划研 究——深圳北站和福田站为例 [J]. 规划师, 2011, 27(10): 23-29.

[52] 季松, 段进. 高铁枢纽地区的规划设计应对策略—以南京南站为例 [J]. 规划师, 2016, 32(3): 68-74.

[53] NOVY J, PETERS D. Chapter 10 railway megaprojects as catalysts for the re-making of post-industrial cities? the case of Stuttgart 21 in Germany[J]. Urban megaprojects: a worldwide view, 2013, 13: 237-262

[54] MONZÓN A, ORTEGA E, LÓPEZ E. Efficiency and spatial equity impacts of high-speed rail extensions in urban areas[J]. Cities, 2013, 30: 18-30.

[55] PUGA D. Agglomeration and cross-border infrastructure[J]. EIB papers, 2008, 13(2): 102-124.

[56] MURAKAMI J. Rail transit technologies, urban regeneration programs, and land value redistributions in the Tokyo Megalopolis Region[C]. Proceedings of the Transportation Research Board 90th Annual Meeting, 2011: 23-27.

[57] HAN J, HAYASHI Y, JIA P, et al. Economic effect of high-speed rail: empirical analysis of Shinkansen's impact on industrial location[J]. Journal of transportation engineering, 2012, 138(12): 1551-1557.

[58] MOULAERT F, SALIN E, WERQUIN T. Euralille large-Scale urban development and social polarization[J]. European urban and regional studies, 2001, 8(2): 145-160.

[59] HAYNES K E. Labor markets and regional transportation improvements: the case of high-speed trains an introduction and review[J]. The annals of regional science, 1997, 31(1): 57-76.

[60] AHLFELDT G. Rail mega-projects in the realm of inter-and intra-city accessibility: evidence and outlooks for Berlin[J]. Built environment, 2012, 38(1): 71-88.

[61] LOUKAITOU-SIDERIS A, HIGGINS H, PIVEN M, et al. Tracks to change or mixed signals? a review of the Anglo-Saxon literature on the economic and spatial impacts of high-speed rail[J]. Transport reviews, 2013, 33(6): 617 633.

[62] LOUKAITOU-SIDERIS A, CUFF D, HIGGINS T, et al. Impact of high speed rail stations on local development: a Delphi survey[J]. Built environment, 2012, 38(1): 51-70.

[63] 林辰辉. 我国高铁枢纽站区开发的影响因素研究 [J]. 国际城市规划, 2011, 26(6): 72-77.

[64] BANISTER D, BERECHMAN Y. Transport investment and the promotion of economic growth[J]. Journal of transport geography, 2001, 9(3): 209-218.

[65] BELLET C. The introduction of the high speed rail and urban restructuring: the case of Spain[J]. City futures, 2009(9): 4-6.

[66] RIBALAYGUA C, GARCIA F. Hsr stations in Europe: new oportunities for urban regeneration[R]. European Regional Science Association, 2011.
[67] TODOROVICH P, SCHNED D, LANE R. High speed rail: international lessons for US policy makers[M]. Cambridge, USA: Lincoln Institute of Land Policy, 2011.

[68] PRIEMUS H. Urban dynamics and transport infrastructure: towards greater synergy[J]. Raiway development, 2008: 15-33.

[69] GARMENDIA M, ROMERO V, UREÑA J M, et al. High-speed rail opportunities around metropolitan regions: Madrid and London[J]. Journal of infrastructure systems, 2012, 18(4): 305-313.

[70] FELIU J. High-speed rail in European medium-sized cities: stakeholders and urban development[J]. Journal of urban planning and development, 2012, 138(4): 293-302.

[71] 林辰辉, 马璇. 中国高铁枢纽站区开发的功能类型与模式 [J]. 城市交通, 2012(5): 41-49.

(本文编辑：许玫) 